\title{
VALVE-PRESERVING REPLACEMENT OF THE ASCENDING AORTA: REMODELING VERSUS REMPLANTATION
}

\author{
H.-J. Schäfers, MD, PhD \\ R. Fries, $\mathrm{MD}^{\mathrm{b}}$ \\ F. Langer, MD \\ N. Nikoloudakis, $\mathrm{MD}^{\mathrm{a}}$ \\ T. Graeter, MD \\ U. Grundmann, $\mathrm{MD}^{\mathrm{c}}$
}

Objective: Aortic valve regurgitation in combination with dilatation of the ascending aorta and root requires a combined procedure to restore valve function and eliminate pathologic dilatation of the proximal aorta. Two techniques have been proposed for this purpose; the aortic root may be either remodeled with an especially configured vascular graft or replaced with reimplantation of the aortic valve within the graft. We have used both techniques depending on the individual pathologic condition of the aortic root. Methods: Of 107 patients undergoing operation for proximal aortic disease between October 1995 and November 1997, 40 patients had morphologically intact aortic valve leaflets in conjunction with dilatation of the aortic root. Of these, 15 patients underwent an operation as a surgical emergency for acute aortic dissection type A. In 29 instances, root remodeling in conjunction with ascending aortic replacement was performed; 11 patients underwent radical replacement of the proximal aorta with reimplantation of the aortic valve. Partial or total arch replacement was performed additionally in 27 of these patients. Other concomitant procedures were coronary artery bypass grafts $(n=11)$ and mitral reconstruction $(n=1)$. Results: Two patients died after repair of acute aortic dissection, for a total operative mortality rate of $5 \%$. No patient died after elective surgery. Aortic valve function could be effectively restored with both techniques. No patient underwent reoperation on the proximal aorta; freedom from aortic regurgitation of grade II or more at 1 year is $88 \%$ with both techniques. Conclusions: Depending on individual root pathologic condition, both the remodeling and the reimplantation techniques appeared to have their individual merits. Both result in adequate restoration of aortic valve function and elimination of pathologic aortic dilatation. ( $J$ Thorac Cardiovasc Surg 1998;116:990-6)
$\mathrm{D}$ ilatation of the ascending aorta occurs as a localized manifestation of a generalized process (eg, congenital connective tissue defect or secondary atherosclerotic degeneration). ${ }^{1,2}$ Commonly the aortic root is also involved in this process, leading to secondary aortic regurgitation despite the presence of morphologically

From the Departments of Thoracic and Cardiovascular Surgery, Cardiology, ${ }^{\mathrm{b}}$ and Anesthesiology, ${ }^{\mathrm{c}}$ University Hospitals, Homburg, Germany.

Received for publication Jan 30, 1998; revisions requested April 22, 1988; revisions received July 2, 1988; accepted for publication July 13, 1988.

Address for reprints: Prof Dr H.-J. Schäfers, Director, Department of Thoracic and Cardiovascular Surgery, University Hospitals, Homburg, 66421 Homburg/Saar, Germany.

Copyright $@ 1998$ by Mosby, Inc.

0022-5223/98 $\$ 5.00+0 \quad \mathbf{1 2 / 1 / 9 3 0 9 0}$ normal valve leaflets. ${ }^{3}$ Regardless of the cause, the spontaneous prognosis is limited primarily either by aortic complications (that is, dissection or rupture) or by congestive heart failure caused by aortic regurgitation. Surgical treatment is directed at relieving both aspects, that is, elimination of the pathologic aortic wall and restoration of the aortic valve function. Composite replacement of the aortic valve and the proximal aorta has become a standard operation with an operative mortality rate of $5 \%$ or less in experienced hands. ${ }^{4-6}$ This, however, exposes the patient to the disadvantages of a prosthetic heart valve. Valve-preserving replacement of the proximal aorta has been advocated as an alternative approach.

Sarsam and Yacoub ${ }^{7}$ have suggested replacement of the ascending aorta and root including the sinuses of Valsalva; by effectively remodeling the aortic root and decreasing 
the diameter of the sinotubular junction, coaptation of the aortic valve leaflets is improved. David and Feindel ${ }^{8}$ developed a technique of complete root replacement, in which a Dacron graft is anchored to the aortoventricular junction and the aortic valve is reimplanted within the graft. Both approaches achieve the 2 goals of a competent aortic valve and elimination of pathologic aortic wall. The individual merits of these 2 techniques have been difficult to judge. Few surgeons have used both approaches, allowing for adequate comparison without major interindividual surgical differences.

It has been our impression that these 2 approaches differ with respect to aggressiveness of root stabilization. We have thus used both techniques in a complimentary fashion. In moderate dilatation of the aortic root (diameter of the sinotubular junction, 3.5 to $5 \mathrm{~cm}$ ), remodeling of the root is used. If aortic root dilatation is severe $(>5 \mathrm{~cm})$ and also involves the aortoventricular junction (>30 mm), aggressive root replacement with reimplantation of the aortic valve within the graft is used. This approach has been used for the correction of preexistent root pathologic condition both in degenerative disease and acute dissection. The early results are reported.

\section{Patients and methods}

Between October 1995 and November 1997, 107 patients underwent an operation for proximal aortic disease in our institution. The underlying diagnosis was aneurysmal dilatation in 73 cases and acute aortic dissection in 29 cases; 5 patients had chronic aortic dissection.

The choice of operative approach was based primarily on the pathologic condition of the aortic valve and root, both in aneurysmal dilatation and acute dissection. If the aortic valve was stenotic or had been replaced previously, composite replacement of aortic valve and ascending aorta was performed. If the aortic valve was morphologically normal and the root was of normal or near normal caliber $(\leq 3.5 \mathrm{~cm})$, supracommissural replacement was chosen. In acute dissection, the root was repaired using gelatine-resorcin-formol (GRF) glue before supracommissural aortic replacement.

If root dilatation was present in conjunction with a morphologically normal aortic valve, valve-preserving root replacement was performed irrespective of the preoperative degree of aortic valve regurgitation. If dilatation was limited to the sinotubular junction, the aorta was replaced including the sinuses of Valsalva, thus remodeling the root. If dilatation was severe, including the aortoventricular junction, or clinical signs of Marfan's syndrome were present, aggressive root replacement with reimplantation of the aortic valve within the vascular graft was performed. In acute dissection, the respective operative steps were performed after application of GRF glue to the dissected aortic wall layers of the root.

Of the total patient population, 40 patients had morphologically normal aortic valve leaflets and significant dilatation of the aortic root. In this subgroup, the patients' ages ranged from 21 to 83 years; 28 patients were men. The underlying disease was annuloaortic ectasia or aneurysmal disease in 23 patients and acute aortic dissection type $\mathrm{A}$ in 15 patients. Two patients had chronic aortic dissection type A. The preoperative degree of aortic valve regurgitation ranged from grade I to grade IV, with a mean of 2.8. In 11 cases significant coronary artery disease was present; in 2 of these cases, coronary artery bypass surgery had been performed previously. Twenty-seven patients had extensive aortic disease involving the aortic arch and requiring concomitant arch replacement. A bicuspid, but morphologically normal, valve was present in 5 cases. The clinical signs of Marfan's syndrome were observed in 3 patients, 1 of whom also had severe mitral regurgitation caused by chordal rupture.

Operative technique. The chest was opened by median sternotomy, and the patient was placed on cardiopulmonary bypass with either the femoral artery or the aortic arch for arterial cannulation. After induction of cardiac arrest by infusion of cardioplegic solution into the coronary ostia, the aortic valve and root were inspected, and the diameters of aortoventricular junction and sinotubular junction were measured. In acute dissection, the wall layers of the aortic root were reconstructed at this point with GRF adhesive (Colle chirurgicale, Cardial, St Etienne, France). The sinuses of Valsalva were excised, leaving approximately 4 to $5 \mathrm{~mm}$ of aortic wall adjacent to the insertion line of the valve leaflets.

For remodeling of the root, a Dacron graft was chosen with a diameter corresponding to that of the aortoventricular junction. The graft was then configured in such a way that the edges conformed to the insertion lines of the aortic valve leaflets. This graft was then sutured to the aortic root with the suture following the insertion line of the valve leaflets. The valve was tested for competence, and, if necessary, additional reconstructive procedures were performed on the valve leaflets. This approach was chosen in all instances of bicuspid aortic valve.

In the presence of a dilated aortoventricular junction or clinical signs of Marfan's syndrome, the aortic valve was mobilized to the aortoventricular junction (ie, the level of the lowest points of the sinuses of Valsalva). A Dacron graft was chosen according to the maximum height of the free edge of the aortic valve leaflets, leaving approximately $30 \%$ to $40 \%$ of leaflet height for coaptation. The graft was then configured to account for the ventricular muscle extension into the commissure between the right and left coronary sinuses. ${ }^{8}$ Transmural mattress sutures were placed along the level of surgical dissection. These sutures were then passed through the graft and tied, anchoring the graft to the aortoventricular junction. The valve was reimplanted within the graft in typical fashion. ${ }^{8,13}$

The coronary ostia were implanted into the graft. The competence of the aortic valve and the absence of suture line bleeding were ascertained by infusion of cardioplegic solution into the graft. If necessary, partial or total arch replacement was performed after hypothermic circulatory arrest. For partial arch replacement, the nasopharyngeal temperature was lowered to $21^{\circ} \mathrm{C}$; for total arch replacement, to $18^{\circ} \mathrm{C}$. Retrograde cerebral perfusion was used only in atherosclerotic aneurysmal disease. 


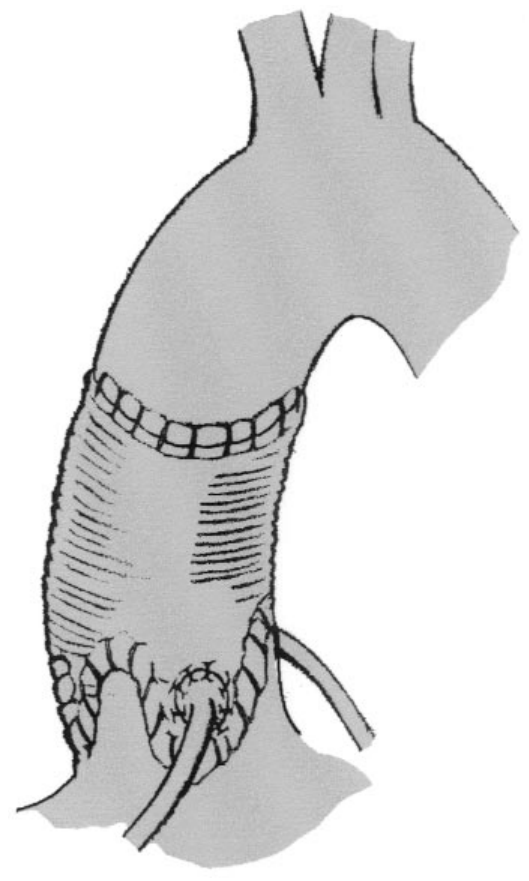

Fig 1. A schematic drawing of the remodeling procedure. A Dacron graft is tailored to conform the shape of the 3 aortic sinuses and then anastomosed to the aortic root.

Adequate function of the aortic valve was ascertained intraoperatively with transesophageal echocardiography after discontinuation of cardiopulmonary bypass. Blood pressure was manipulated pharmacologically to maintain a diastolic pressure of $70 \mathrm{~mm} \mathrm{Hg}$ at the time of echocardiography. Postoperatively, echocardiography was performed after 7 days and after 3, 6, 12, 18, and 24 months in transthoracic fashion with the patient in a resting position (HDI 3.000; phased array, 3.24-1.75 MHz; ATL [Advanced Technology Laboratories], Bothell, Wash). A semiquantitative assessment of the degree of aortic valve regurgitation was performed with intensity and slope of regurgitation signal and relative size of the regurgitation jet in relation to the diameter of the left ventricular outflow tract. ${ }^{9,10}$ Systolic flow gradients were determined, and the peak systolic gradient was recorded. ${ }^{11}$ All echocardiographic studies were performed by the same investigator.

Statistical methods. All data were reviewed retrospectively. A comparative analysis was performed between the 2 different patient groups. Differences were analyzed for discrete variables with the $\chi^{2}$ test; the Mann-Whitney $U$ test was used for continuous variables.

\section{Results}

According to the decision criteria outlined here, a remodeling procedure was performed in 29 patients; 11 patients underwent root replacement with reimplanta-

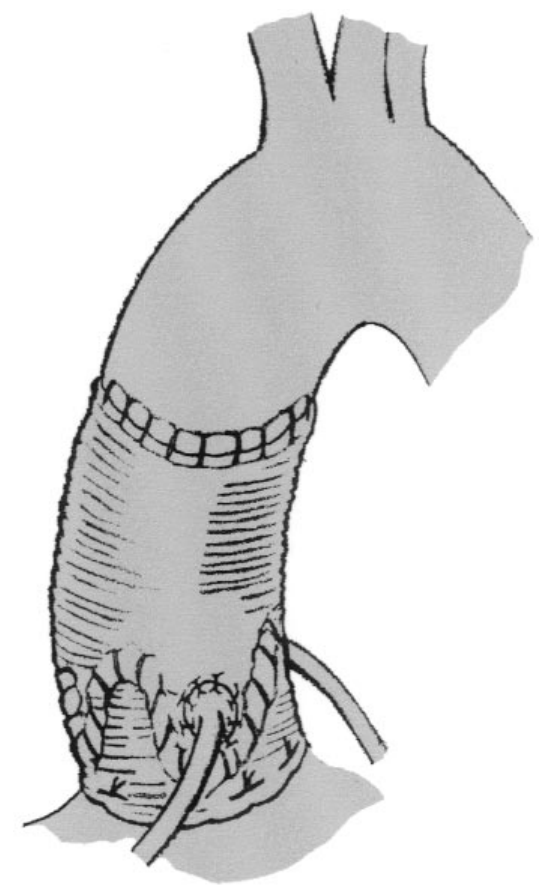

Fig 2. A schematic drawing of the reimplantation procedure. After mobilization of the aortic root, a Dacron graft is anchored to the aortoventricular junction. The native aortic valve is then resuspended within the vascular graft.

tion of the native aortic valve as originally described by David and Feindel. ${ }^{8}$

Partial replacement of the aortic arch was performed for acute dissection or aneurysmal arch disease in 23 patients; 4 patients underwent total arch replacement with elephant trunk extension for mega-aortic syndrome $(n=2)$ or extensive dissection with multiple arch entries $(\mathrm{n}=2)$. Concomitant procedures were coronary artery bypass grafts $(n=10)$ and mitral reconstruction $(n=1)$.

One patient in the remodeling group underwent reexploration for surgical bleeding. There was no instance of a perioperative aortoventricular conduction disturbance.

Two patients died after the repair of acute aortic dissection type A. The cause of death was unrelated to the choice of root procedure in both instances. One of these patients died of massive pulmonary embolism after root remodeling and coronary artery bypass operation for acute dissection with severe obesity as a risk factor for thrombosis. The patient experienced cardiac arrest and died on the regular ward 2 weeks after the operation. The second patient underwent a root remodeling procedure for acute dissection; the operation was performed after cardiopulmonary resuscitation for cardiac arrest during induction of anesthesia. After the opera- 


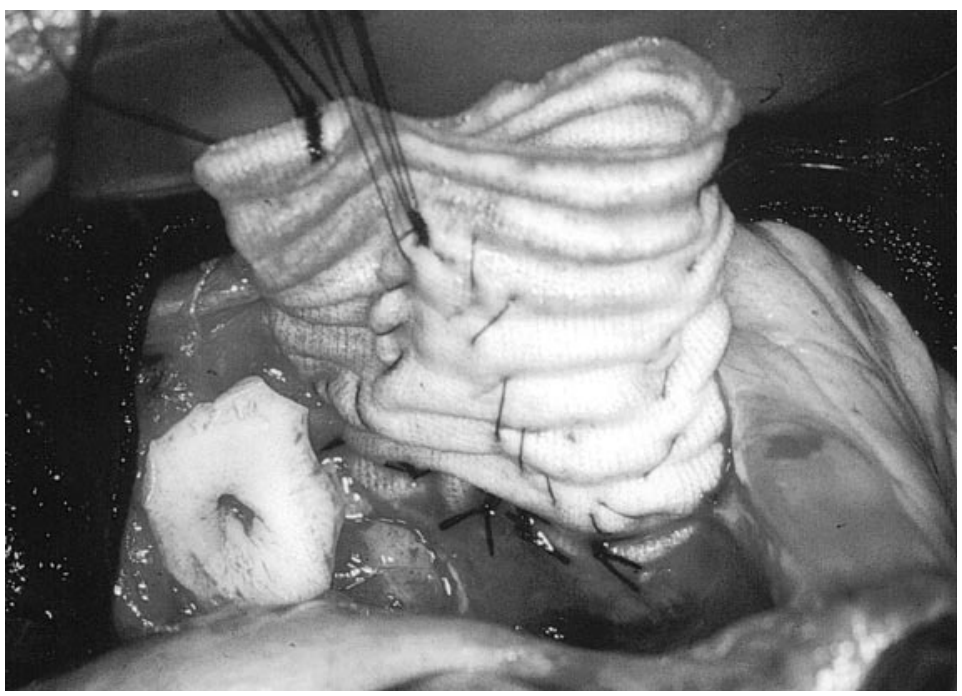

Fig 3. Operative photograph of reimplantation procedure. The tubular graft encloses the aortic root to the level of the aortoventricular junction, and the valve is reimplanted within the graft. The suture line that follows the insertion of the aortic valve leaflet can be seen.

Table I. Preoperative data in the patients undergoing valve-preserving operations on the ascending aorta

\begin{tabular}{lccl}
\hline & Remodeling & Resuspension & $\mathrm{P}$ \\
\hline Patients (No.) & 29 & 11 & \\
Sex (M/F) & $18 / 11$ & $10 / 1$ & .1 \\
Age (yr) & $64 \pm 12$ & $49 \pm 17$ & .01 \\
NYHA & $2.9 \pm 1.3$ & $3.1 \pm 0.8$ & .9 \\
CAD (No.) & $10 / 29$ & $1 / 11$ & .1 \\
1VD (No.) & 2 & 1 & .8 \\
2VD (No.) & 3 & - & .3 \\
3VD (No.) & 5 & - & .1 \\
LVEF (\%) & $64 \pm 13$ & $67 \pm 3$ & .9 \\
Grade AI (mean) & $2.7 \pm 0.4$ & $2.8 \pm 0.5$ & .6 \\
Biscuspid aortic valve (No.) & 5 & 0 & .1 \\
AADA (No.) & 12 & 3 & .4 \\
\hline
\end{tabular}

$M$, Male; $F$, female; NYHA, New York Heart Association; $C A D$, coronary artery disease; $1 V D$, single vessel disease; $2 V D$, double vessel disease; $3 V D$, triple vessel disease; $L V E F$, left ventricular ejection fraction; $A I$, aortic insufficiency determined by Doppler echocardiography graded I to IV; $A A D A$, acute aortic dissection type A.

tion, diffuse cerebral infarction was documented. The patient died primarily of a cerebral cause.

During follow-up, no evidence for valve endocarditis was observed; no patient required reoperation on the proximal aorta. Two patients underwent scheduled distal aortic replacement as completion of staged replacement in mega-aortic syndrome.

Comparing the remodeling to the reimplantation procedure, there was a higher percentage of operations for acute dissection performed on an emergency basis in the remodeling group. In this group the patients tended
Table II. Perioperative data in 40 patients undergoing valve-preserving replacement of the proximal aorta

\begin{tabular}{lccl}
\hline & Remodeling & Reimplantation & $\mathrm{P}$ \\
\hline $\begin{array}{l}\text { Intraoperative data } \\
\quad \text { Aortoventricular diameter }\end{array}$ & $25 \pm 2$ & $33 \pm 1$ & .04 \\
$\quad(\mathrm{~mm})$ & & & \\
$\quad$ Sinotubular diameter (mm) & $43 \pm 1$ & $56 \pm 9$ & .01 \\
$\quad \begin{array}{l}\text { Maximum ascending aortic } \\
\quad \text { diameter (mm) }\end{array}$ & $63 \pm 11$ & $65 \pm 8$ & .5 \\
$\quad$ Mycardial ischemia (min) & $87 \pm 25$ & $112 \pm 32$ & .03 \\
$\quad$ Cardiopulmonary bypass & $133 \pm 38$ & $145 \pm 23$ & .2 \\
$\quad$ (min) & & & \\
$\quad$ Circulatory arrest (min) & $18 \pm 10$ & $19 \pm 12$ & .8 \\
Concomitant procedures (No.) & & & \\
$\quad$ Partial arch replacement & 19 & 4 & .1 \\
$\quad$ Total arch replacement & 3 & 1 & .9 \\
$\quad$ CABG & 10 & 1 & .1 \\
Mitral reconstruction & 0 & 1 & .1 \\
\hline
\end{tabular}

$C A B G$, Coronary artery bypass graft.

to be older ( $P=$ not significant $)$, and aortic crossclamp times were significantly shorter $(87 \pm 25$ minutes versus $112 \pm 32$ minutes; $P=.03$ ). A higher proportion of patients in the remodeling group underwent concomitant arch replacement ( 22 of 29 patients versus 5 of 11 patients) and coronary artery bypass surgery (10 of 29 patients versus 1 of 11 patients). There was no difference in cardiopulmonary bypass times $(133 \pm 38 \mathrm{~min}-$ utes versus $145 \pm 23$ minutes). 


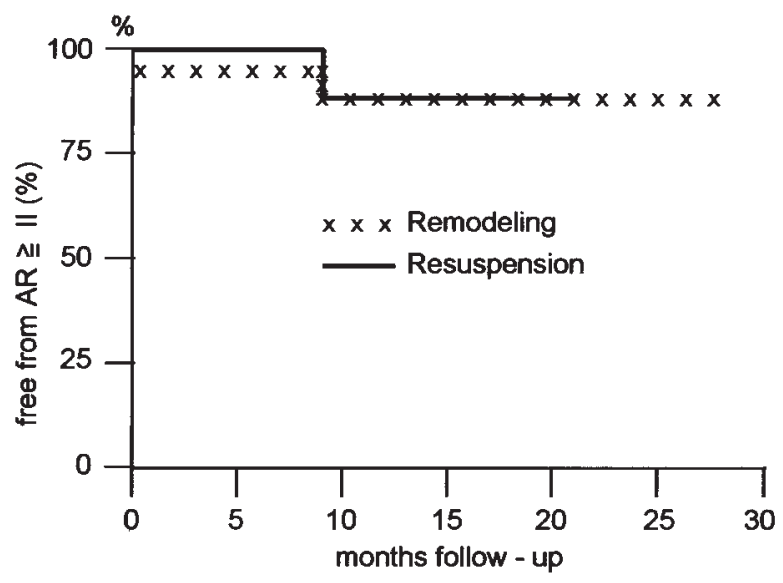

Fig 4. Actuarial freedom from postoperative aortic regurgitation grade II or higher in reimplantation and remodeling techniques.

The preoperative degree of aortic regurgitation, determined semiquantitatively by Doppler echocardiography, was almost identical in the 2 groups $(2.7 \pm 0.4$ versus $2.8 \pm 0.3$ ). Postoperatively the degree of aortic regurgitation was reduced in almost identical fashion $(0.5 \pm 0.6$ versus $0.5 \pm 0.7)$. Most patients had either no or minimal aortic regurgitation. Two patients in the remodeling group had aortic regurgitation grade II after the operation. The patients had been reduced from preoperative grade III to IV; intraoperatively, the leaflets had shown mild degenerative changes. One patient in the reimplantation group experienced the development of aortic regurgitation grade II in the course of the first 18 months. Peak systolic gradients across the aortic valve were comparable in both groups.

After a mean follow-up of 11 months, freedom from reoperation on the aortic valve is $100 \%$. Actuarial freedom from aortic regurgitation grade II or greater 1 year after the operation is $88 \%$.

\section{Discussion}

To avoid the disadvantages of prosthetic heart valves, 2 different valve-preserving operations for aortic root dilatation have been proposed. Sarsam and Yacoub ${ }^{7}$ have propagated a remodeling procedure (ie, replacement of the aortic root including the sinuses of Valsalva). They maintain that this operation is adequate to avoid secondary dilatation of the aortic root and still to preserve or restore aortic valve function. David and Feindel ${ }^{8}$ have pointed out that, in extensive root dilatation, not only the sinuses of Valsalva are dilated but also the fibrous portions of the root inferior to the valve insertion line (ie, fibrous trigone and membranous sep-
Table III. Echocardiographic data comparing degree of aortic insufficiency and peak systolic gradient of remodeling and reimplantation technique

\begin{tabular}{lllll}
\hline & $\begin{array}{c}\text { Before } \\
\text { operation }\end{array}$ & $\begin{array}{c}\text { After } \\
\text { operation }\end{array}$ & \multicolumn{1}{c}{6 mo } & 12 mo \\
\hline AI (grade 0-4) & & & & \\
$\quad$ Mean & $2.9 \pm 0.5$ & $0.5 \pm 0.6$ & $0.5 \pm 0.6$ & $0.5 \pm 0.7$ \\
$\quad \begin{array}{l}\text { Range } \\
\text { Remodeling } \\
\text { Peak gradient } \\
\quad \text { (mm Hg) }\end{array}$ & & $0-2$ & $0-2$ & $0-2$ \\
$\quad$ Mean & & & & \\
$\quad$ Range & & $1.4-14.3$ & $2.7-15.8$ & $3.2-15.0$ \\
AI (grade 0-4) & & & & \\
$\quad$ Mean & $3.0 \pm 0.5$ & $0.5 \pm 0.2$ & $0.6 \pm 0.8$ & $0.6 \pm 0.9$ \\
$\quad$ Range & $2-4$ & $0-1$ & $0-1$ & $0-2$ \\
$\begin{array}{c}\text { Reimplantation } \\
\text { Peak gradient } \\
\text { (mm Hg) }\end{array}$ & & & & \\
$\quad \begin{array}{l}\text { Mean } \\
\text { Range }\end{array}$ & & $4.9 \pm 2.1$ & $5.0 \pm 2.2$ & $4.8 \pm 2.4$ \\
\hline
\end{tabular}

$A I$, Aortic insufficiency.

tum). To correct the root also at this level, they have proposed mobilization of the root, anchoring a Dacron graft to the aortoventricular junction, and reimplantation of the aortic valve within the vascular graft. ${ }^{8} \mathrm{~A}$ judgment of the relative merits of these 2 procedures is hampered by the fact that few long-term data have been published. This situation is made even more difficult by the fact that David ${ }^{12}$ has proposed a new modification of the original operative technique.

It has been our feeling that both approaches have merits and probably should not be chosen in a competitive, but rather complimentary, fashion. Clinical observations suggest that in many patients with atherosclerotic-degenerative aneurysm of ascending aorta and root, the aortoventricular junction is of normal diameter. ${ }^{12,13}$ Remodeling of the aortic root appears to completely eliminate the pathologic process. In socalled annulo-aortic ectasia, and particularly in Marfan's syndrome, however, root dilatation commonly extends into the aortoventricular junction. Although the risk of secondary dilatation of fibrous trigone and membranous septum after root remodeling is difficult to judge at this time, the friability of the fibrous tissue in these areas indicates to us that additional support appears advisable. We have therefore chosen remodeling for root dilatation in the presence of normal aortoventricular junction and aggressive root replacement with valve reimplantation in root dilatation with a dilated aortoventricular junction including Marfan's syndrome. 
Functionally, both approaches appear to be associated with similar results. With respect to the competence of the aortic valve, the results in our patient population confirm the positive results of Sarsam and Yacoub, ${ }^{7}$ David and Feindel, ${ }^{8}$ and David ${ }^{12}$ and of the previous experience of the primary author. ${ }^{13,14}$ Apparently there was no difference with respect to restoration of aortic valve function in the 2 groups. The percentage of patients free from aortic regurgitation greater than stage I was comparable.

Interestingly, the maximum systolic gradients measured in these reconstructed valves were essentially physiologic in all patients. Thus the hemodynamic performance of the reconstructed aortic valve is superior to that of any prosthetic heart valve. Currently, stentless bioprosthesis appear to have the best hemodynamic performance resulting in almost physiologic unloading of the left ventricle. Nonetheless, the mean systolic gradients measured by Doppler echocardiography in these valves range from 7 to $20 \mathrm{~mm} \mathrm{Hg.}{ }^{15-17}$ In our reconstructed tricuspid valves the peak gradients were measured around $5 \mathrm{~mm} \mathrm{Hg}$ in both groups and thus physiologic. Similar absence of systolic gradients can otherwise only be expected in root replacement with either homografts or autografts. ${ }^{18}$ Even in reconstructed bicuspid valves the peak gradients were less than 10 $\mathrm{mm} \mathrm{Hg}$ and thus comparable with or superior to those seen in stentless biografts. It remains to be investigated whether the hemodynamic performance of these valves will also by physiologic under conditions of exercise.

It has been hypothesized that reimplantation of the aortic valve alters the geometry of the sinuses of Valsalva and inhibits the dynamic component of the aortic root during the cardiac circle. ${ }^{19}$ Although the remodeling technique appears to result in a lesser degree of alteration of root geometry and function, the clinical significance of these considerations is at this time unclear. The original reimplantation technique, ${ }^{8}$ however, appears to provide more complete external stabilization of the fibrous portions of the aortic root compared with a recent modification. ${ }^{12}$

Both approaches have not been associated with thromboembolic complications and therefore do not require anticoagulation with its associated hemorrhagic complications. In analogy with the experience made in reconstruction of the mitral valve, the risk of valve endocarditis can be expected to be lower than that of a prosthetic heart valve.

Both techniques apparently result in comparable restoration of aortic valve function, but in the presence of a bicuspid aortic valve, the remodeling approach appears to be superior to reimplantation. In the 5 patients in whom a bicuspid valve was reconstructed and the aortic root remodeled, excellent valve function was accomplished. Although we have never attempted reimplantation in the presence of a bicuspid aortic valve, we anticipate significant problems in achieving a perfect root and valve geometry with this anatomic variant.

Crossclamp times and cardiopulmonary bypass time were shorter in the remodeling group despite more extensive aortic surgery. Placement of additional sutures in the aortic root for suture line bleeding, however, outweighed the apparent advantage as a less complex operation. This was particularly apparent in patients undergoing an operation for acute dissection. Hemostasis in the aortic root in these patients was easier to achieve by the reimplantation technique.

In 5 patients limited leaflet prolapse persisted after completion of the respective root procedure. On the basis of previous experience, ${ }^{14}$ we have corrected leaflet prolapse using established techniques ${ }^{20-22}$ to achieve near-perfect geometry of the aortic root. In a previous series, ${ }^{14}$ it became apparent that perfect, or at least near-perfect, geometry of the aortic valve was an important prerequisite for medium-term valve competence with the reimplantation approach. In 2 patients of the previous series only trivial incompetence had been observed at the initial operation despite prolapse of 1 valve leaflet. Within 12 to 18 months, severe regurgitation led to reoperation. It is at present unclear whether root asymmetry can lead to valve degeneration in root remodeling also or whether this approach is more "forgiving" than the reimplantation technique. Although there are no long-term data on the fate of resuspended aortic valves that have undergone additional reconstructive procedures on the valve leaflets, our current medium-term observations suggest that these valves remain stable.

It is still too early to determine the long-term fate of the native aortic valve after root reconstruction, but we anticipate good long-term function at least in those instances in which perfect valve geometry was achieved and either trivial or no aortic valve incompetence is seen within the first 2 years. Further follow-up will be needed to determine the long-term fate and risk of the reoperation. If reintervention becomes necessary, a valve prosthesis can easily be implanted into the aortic root graft.

We therefore conclude that valve-preserving operations can be performed on the ascending aorta as part of major aortic interventions with low morbidity and mortality rates. Both remodeling of the aortic root and reimplantation of the valve within a vascular graft lead to comparable early and medium-term results. Both 
procedures appear to result in good restoration of aortic valve function.

\section{REFERENCES}

1. Halloran BG, Davis, VA, McManus BM, Lynch TG, Baxter BT. Localization of aortic disease is associated with intrinsic differences in aortic structure. J Surg Res 1995;59:17-22.

2. Roman MJ, Rosen SE, Kramer-Fox R, Devereux RB. Prognostic significance of the pattern of aortic root dilatation in the Marfan syndrome. J Am Coll Cardiol 1993;22:1470-6.

3. Becker AE. Surgical and pathological anatomy of the aortic valve and root. Operative Techniques Card Thorac Surg 1996;1:3-14.

4. Borst HG, Laas J. Surgical treatment of thoracic aortic aneurysms. Adv Cardiac Surg 1993;4:47-87.

5. Bachet J, Termingnon JL, Goutot B, Dreyfus G, Piquois A, Brodaty D, et al. Aortic root replacement with a composite graft: factors influencing immediate and long-term results. Eur J Cardiothoracic Surg 1996;10:207-13.

6. Gott VL, Gillinov AM, Pyeritz RE, Cameron DE, Reitz BA, Greene PS, et al. Aortic root replacement: risk factor analysis of a seventeen-year experience with 270 patients. J Thorac Cardiovasc Surg 1995;109:536-44.

7. Sarsam MA, Yacoub M. Remodeling of the aortic valve annulus. J Thorac Cardiovasc Surg 1993;105:435-8.

8. David TE, Feindel CM. An aortic valve-sparing operation for patients with aortic incompetence and aneurysm of the ascending aorta. J Thorac Cardiovasc Surg 1992;103:617-22.

9. Galassi AR, Nihoyannopoulos P, Pupita G, Odwadara H, Crea F, McKenna WJ. Assessment of color flow imaging in the grading of valvular regurgitation. Eur Heart J 1990;11:1101-8.

10. Masuyama TK, Kodama AK, Kitabatake A, Nanto S, Sato H, Uematsu M, et al. Non-invasive evaluation of aortic regurgitation by continuous-wave Doppler echocardiography. Circulation 1987;73:460-6.
11. Skjerpe T, Hegrenaes L, Hatle L. Noninvasive estimation of valve area in patients with aortic stenosis by Doppler ultrasound and two-dimensional echocardiography. Circulation 1985;72:810-5.

12. David TE. Remodeling the aortic root and preservation of the native aortic valve. Operative Techniques Card Thorac Surg 1996;1:44-56.

13. Schäfers HJ, Borst HG. Valve-sparing operation in aortic root ectasis. Operative Techniques Card Thorac Surg 1996;1:38-43.

14. Pethig K, Schäfers HJ, Borst HG. Functional results after aortic valve repair in aortic root ectasia. J Heart Valve Dis 1996;5:247-50.

15. Achtelik M, Pethig K, Schäfers HJ, Borst HG. Stentless aortic valve replacement with Biocor prosthesis: indication and outcome. J Heart Valve Dis 1995;5:314-6.

16. Westaby S, Amarasena N, Long V, Prothero A, Chandima Amarasena GA, Banning AP, et al. Time-related hemodynamic changes after aortic replacement with the Freestyle stentless xenograft. Ann Thorac Surg 1995;60:1633-9.

17. Sintek CF, Fletcher AD, Khonsari S. Stentless procine aortic root: Valve of choice for the elderly patient with small aortic root? J Thorac Cardiovasc Surg 1995;109:871-6.

18. Skillington PD, Grigg LE. Insertion of the pulmonary autograft as an inclusion cylinder-modifications to existing techniques. J Heart Valve Dis 1995;4:374-8.

19. Brewer RJ, Deck JD, Capat ID, Nolan SP. The dynamic aortic root: its role in aortic valve function. J Thorac Cardiovasc Surg 1976;72:413-7.

20. Cohn LH, Couper GS, Aranke SF, Rizzo RJ, Kinchala NM, Collins JJ. Long-term results of mitral valve reconstruction for regurgitation of myxomatous mitral valve. J Thorac Cardiovasc Surg 1994;107:145-50.

21. Trusler GA, Moes CAF, Kidd BSL. Repair of ventricular septal defect with aortic insufficiency. J Thorac Cardiovasc Surg 1973;66:394-8.

22. Cosgrove DM, Rosenkranz ER, Hendren WG, Bartlett JC, Stewart WJ. Valvuloplasty for aortic insufficiency. J Thorac Cardiovasc Surg 1991;102:571-7. 